\title{
Characterization of modulated time-of-flight range image sensors
}

\author{
Andrew D. Payne*a, Adrian A. Dorrington ${ }^{\mathrm{a}}$, Michael J. Cree ${ }^{\mathrm{a}}$, Dale A. Carnegie ${ }^{\mathrm{b}}$ \\ ${ }^{a}$ Dept. of Engineering, University of Waikato, Hamilton, New Zealand; \\ ${ }^{\mathrm{b}}$ School of Chemical and Physical Sciences, Victoria University of Wellington, New Zealand
}

\begin{abstract}
A number of full field image sensors have been developed that are capable of simultaneously measuring intensity and distance (range) for every pixel in a given scene using an indirect time-of-flight measurement technique. A light source is intensity modulated at a frequency between $10-100 \mathrm{MHz}$, and an image sensor is modulated at the same frequency, synchronously sampling light reflected from objects in the scene (homodyne detection). The time of flight is manifested as a phase shift in the illumination modulation envelope, which can be determined from the sampled data simultaneously for each pixel in the scene. This paper presents a method of characterizing the high frequency modulation response of these image sensors, using a pico-second laser pulser. The characterization results allow the optimal operating parameters, such as the modulation frequency, to be identified in order to maximize the range measurement precision for a given sensor. A number of potential sources of error exist when using these sensors, including deficiencies in the modulation waveform shape, duty cycle, or phase, resulting in contamination of the resultant range data. From the characterization data these parameters can be identified and compensated for by modifying the sensor hardware or through post processing of the acquired range measurements.
\end{abstract}

Keywords: 3D imaging, 3D sensor, modulated image sensor, range imaging, time-of-flight.

\section{INTRODUCTION}

Solid-state range imaging is an emerging technology with many potential applications in the growing field of computer based machine and robotic vision. Range imaging systems generate a digital photograph like output with distance information contained in every pixel, providing the capability to measure the shape, size, and location of objects in the scene. This provides many advantages over traditional imaging for machine vision applications because it is much easier for a computer to accurately identify and locate objects using range data. Potential applications are numerous and include computer vision for mobile robotics, production line quality assurance, computerized safety and security, obstacle identification and avoidance for automotive applications, gesture recognition for computer input or gaming, and 3D special effects in television/movies.

Range imaging systems determine distance from an indirect time of flight measurement, using an amplitude modulated illumination source and a specialized modulated image sensor, where the distance measurement performance is highly dependent on the high speed operation of these components. Traditionally, performance is evaluated by characterizing the entire range imaging system ${ }^{1}$, comprising the illumination source, optics and image sensor, to measure the resultant systematic and random errors. This approach is useful for calibration purposes, but obscures the underlying factors responsible for these errors which limit the measurement precision and accuracy. By measuring the response of the illumination source and modulated image sensor individually, these factors can be investigated in greater detail, potentially allowing restrictive characteristics to be identified and optimized.

The illumination source (typically consisting of infrared LEDs modulated at tens of MHz) can easily be measured with commonly available equipment. A photodiode and oscilloscope provide a means of measuring the high frequency amplitude modulation, while a laser power meter allows the mean output power level to be determined. However, in comparison a more complex approach is required to characterize the high frequency response of each pixel within the modulated image sensor. A technique is demonstrated here that uses a pico-second pulsed laser to optically probe the

*a.payne@waikato.ac.nz; phone +64 7838 4841; fax +64 78384835

Three-Dimensional Imaging Metrology, edited by J. Angelo Beraldin, Geraldine S. Cheok, Michael McCarthy, Ulrich Neuschaefer-Rube, Proc. of SPIE-IS\&T Electronic Imaging, SPIE Vol. 7239, 723904

(c) 2008 SPIE-IS\&T · CCC code: 0277-786X/08/\$18 - doi: 10.1117/12.806007 
modulated image sensor, providing a measurement at one instant in the sensor modulation period. By continually altering the timing of the laser pulse and repeating the capture process, a complete profile of the high frequency image sensor modulation can be constructed. The measured response provides the opportunity to identify the optimal operating parameters for the image sensor to maximize range measurement precision, and to identify and compensate for deficiencies in the modulation profile that can lead to systematic errors which in turn reduce measurement accuracy.

\section{BACKGROUND}

\subsection{Measuring time-of-flight}

Range imaging systems use an indirect time-of-flight measurement to determine distance to objects in the scene. The scene is flood illuminated by an intensity modulated source, and the light is reflected back toward a specialized image sensor. The return flight time (from the light source to the object and back to the sensor) produces a phase shift in the illumination modulation envelope proportional to the object distance. The image sensor measures this phase shift within each pixel by synchronously sampling the incoming light at a frequency identical to that of the light source (homodyne detection). Typically four equally spaced measurements of the illumination waveform $\left(\mathrm{A}_{1}\right.$ to $\left.\mathrm{A}_{3}\right)$ are taken by shifting the phase of the sensor modulation signal by $\pi / 2 \operatorname{rad}\left(90^{\circ}\right)$, allowing the three unknown variables amplitude $A$, offset $B$, and phase $\varphi$ to be determined using equations (1) to (3). The offset value $B$ is a combination of background (ambient) light $A_{\text {amb }}$ and an offset $A_{\text {sig }}$ from the illumination source and sensor modulation waveforms as illustrated in Fig. 1. Finally, distance $d$ to each pixel is determined using (4), where $c$ is the speed of light, and $f_{\text {mod }}$ is the modulation frequency of the illumination source and the sensor.

$$
\begin{gathered}
A=\frac{\sqrt{\left(A_{0}-A_{2}\right)^{2}+\left(A_{1}-A_{3}\right)^{2}}}{2} \\
B=\frac{A_{0}+A_{1}+A_{2}+A_{3}}{4} \\
\varphi=\tan ^{-1}\left(\frac{A_{0}-A_{2}}{A_{1}-A_{3}}\right) . \\
d=\frac{c \varphi}{4 \pi f_{\bmod }}
\end{gathered}
$$

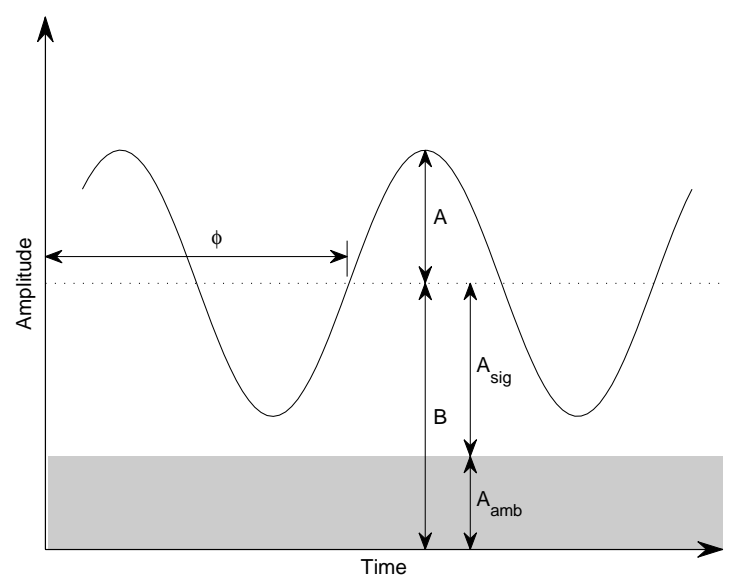

Fig. 1. Measured modulation signal illustrating the three unknown variables: amplitude $A$, offset $B$, and phase $\varphi$. Ambient light contributes a DC offset, illustrated by the shaded region. 


\subsection{Range measurement precision}

Optical measurement systems are fundamentally limited by photon shot noise, in this instance limiting the range measurement standard deviation $\sigma_{\mathrm{R}}$ as given by ${ }^{2}$

$$
\sigma_{R}=\frac{c}{4 \pi f_{\text {mod }} \sqrt{2}} \cdot \frac{\sqrt{B}}{c_{\text {demod }} \cdot A_{\text {sig }}}
$$

where $c_{\text {demod }}$ is known as the demodulation contrast, $A_{\text {sig }}$ is the mean signal component resulting from the illumination source, and $B$ is the total signal offset due to both $A_{\text {sig }}$ and background illumination $A_{\text {amb }}$ as illustrated in Fig. 1. The waveform modulation depth, or demodulation contrast, $c_{\text {demod }}$ is calculated for a sine wave as

$$
c_{\text {demod }}=\frac{A}{A_{\text {sig }}}
$$

i.e. the signal amplitude $A$ divided by the signal offset $A_{\text {sig }}$ in the absence of ambient light, with a value between zero and one.

From (5) it is apparent that there are three mechanisms available to improve range measurement precision:

- Decrease the ambient light level relative to the signal illumination power $\left(\sqrt{ } B / A_{\text {sig }}\right)$.

- Increase the modulation frequency $f_{\text {mod }}$.

- Increase the demodulation contrast $c_{\text {demod }}$.

The first approach of decreasing the ambient light level relative to the active illumination signal is the most straightforward, and is implemented on most systems. By placing an optical filter in front of the image sensor, only light at a wavelength matching that used by the illumination source reaches the sensor, and a significant portion of ambient light $A_{a m b}$ is rejected. The emitted optical power can also be increased, for example by adding more light emitting diodes (LEDs) to the system, but there are practical limitations to this approach such as eye safety constraints. Increasing the optical power is likely to also create a corresponding increase in size, electrical power consumption, and associated heat dissipation problems. It is assumed here that these options have already been realized for a given system, and focus is applied on improving measurement precision by alternate means.

The second option is to operate the sensor and illumination source at higher speeds, as the standard deviation of the range measurement is inversely proportional to the modulation frequency $f_{\text {mod }}$ as given by (5). It is therefore desirable to operate the system at as high frequency as possible, as doubling $f_{\text {mod }}$ will halve the range measurement errors. However, in practice the maximum operating frequency is also constrained by the electronic bandwidths of the illumination source and the modulated image sensor. By measuring the modulation response of the illumination source and image sensor at various frequencies it is possible to select an optimal operating frequency within these bandwidth constraints, and also identify limiting components within the system.

Finally, the received signal modulation depth or demodulation contrast $c_{\text {demod }}$ also plays an important part in the range measurement precision. A high value is achieved by ensuring that the illumination and image sensor modulation waveforms turn completely off and then completely on during each modulation cycle, as opposed to that illustrated in Fig. 1 where a DC offset exists and the waveform cannot go to zero even in the absence of ambient light. The demodulation contrast is proportional to the individual modulation depths of the illumination and the image sensor waveforms, $m_{\text {Illumination }}$ and $m_{\text {Sensor }}$ respectively, as given by (7), therefore it is important to maximize these values to achieve high measurement precision. The modulation depth is calculated in the same manner as $c_{d e m o d}$, by taking the signal amplitude divided by the DC offset, although for non-sinusoidal modulation the shape of the waveform also influences the modulation depth value and is discussed further in section 4.1.

$$
c_{\text {demod }}=\frac{m_{\text {Illumination }} m_{\text {Sensor }}}{2}
$$

The demodulation contrast $c_{\text {demod }}$ and modulation frequency $f_{\text {mod }}$ are also dependent on one another. This is evident in the fact that the modulation amplitude $A$ decreases with increasing frequency due to illumination source and image sensor 
modulation bandwidth limitations, while the offset $A_{\text {sig }}$ remains constant. For a given illumination source or image sensor an optimal operating frequency exists that maximizes this tradeoff between $c_{\text {demod }}$ and $f_{\text {mod }}$.

\subsection{Range measurement accuracy}

In addition to maximizing range measurement precision, it is also important to achieve high measurement accuracy. Systematic errors affecting measurement accuracy can be broken into two categories: imaging distortions and systematic range (phase) measurement errors. Imaging distortions are a result of the imaging optics (e.g. the sensor lens) producing radial and other related distortions. These distortions can be calibrated using the same techniques applied to standard 2D cameras, independently of the range measurement data ${ }^{3}$. Range measurement errors can result from a variety of different sources, including multiple optical paths existing between the illumination source and the image sensor pixel, inhomogeneous sensor modulation, and errors in the phase measurement determined using (3). Light reaching the image sensor from multiple paths are averaged together, distorting the resultant range measurement. This can occur for example when an array of LEDs is used, where light from different illumination sources is reflected by an object and captured by a single pixel location. Similarly multiple reflections can be superimposed at a single pixel location from different objects in the scene (known as mixed pixels), or from reflections between the image sensor and the lens ${ }^{4}$. This effect is highly dependent on the configuration of the system (particularly the illumination source and optics), and also on the layout of the scene.

In contrast, inhomogeneous sensor modulation and phase measurement errors are effects that are independent of the scene being measured. Ideally all pixels in the image sensor should be modulated identically with the same frequency, phase, and amplitude. In practice it is possible that, in particular, the phase of the modulation signal may vary across the sensor due to electronic propagation delays between pixels. This creates a pixel location dependent offset in the resultant range measurement. By measuring this fixed delay it is possible to apply a correction to the range measurements to mitigate this error.

The range measurement is determined by taking four equally spaced samples of the incoming waveform, allowing the phase to be calculated using (3). If the illumination or image sensor modulation waveform is not sinusoidal, higher frequency harmonics will exist in the modulation waveform that will cause aliasing during this sampling process. In particular, odd order harmonics are aliased onto the fundamental frequency which contaminate the phase measurement, resulting in a non-linear range output ${ }^{5}$. The shapes of the modulation waveforms therefore have a significant impact on the measurement accuracy. This error is typically minimized through the use of calibration routines ${ }^{1}$, although an alternative method of cancelling the harmonic content during sampling has also recently been demonstrated ${ }^{6}$.

\section{MODULATED IMAGE SENSOR CHARACTERIZATION}

A simplified structure of a pixel within a modulated range image sensor is shown in Fig. 2. Pixel operation consists of converting the incoming light into electrons which are then separated in two channels 1 and 2 by a high frequency modulation input signal. Each channel is integrated over a large number of pulses to provide a high signal to noise ratio (SNR) before the resultant voltages are subtracted from one another and readout from the pixel. The subtraction stage is used to remove any common offset voltage from the two channels that may exist, for example due to uncorrelated (ambient) light reaching the sensor, while effectively doubling the output signal amplitude $A$.

The high speed switching stage within the pixel is of primary interest as the resulting range measurement precision and accuracy is dependent on the image sensor modulation operation as outlined in section 2. The pixel modulation transfer function is unknown; therefore the internal sensor modulation waveform cannot be determined from measurements of the external electrical modulation input signal. Similarly, the pixel output $\mathrm{V}_{\text {out }}$ cannot be used to directly measure the high frequency modulation due to the slow integration and readout stages, and an internal electrical measurement within the pixel is not possible. 


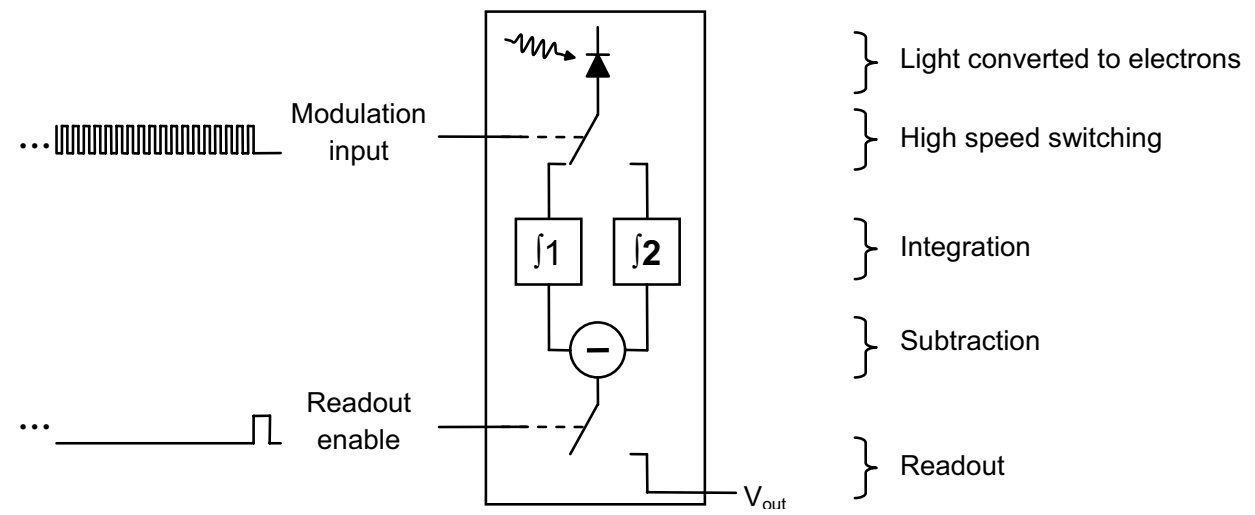

Fig. 2. Basic structure of a modulated pixel.

To overcome these limitations, an optical method can be used to measure the modulation response using a configuration similar to that described in ${ }^{7}$, illustrated in Fig. 3. A field programmable gate array (FPGA) generates the modulation signal (and other auxiliary signals) to control the image sensor, and also generates a second trigger signal at a frequency identical to the modulation signal. The trigger signal is connected to a laser pulser that generates short picosecond duration bursts of light. The laser light is coupled through an optical fiber to produce a circular output (as the raw output from the laser diode pulser is elliptical), and then through a diffuser to provide homogenous illumination of the image sensor.

The short optical laser pulse samples (or probes) the image sensor modulation waveform as shown on the right hand side of Fig. 3, where the identical trigger and modulation frequencies are used to sum together a large number of pulses within the integration stage of the pixel to produce high SNR. At the end of the integration period each pixel is read out from the image sensor, where the resulting output voltage is proportional to the probed point within the modulation waveform. The FPGA then adds a small phase shift to the laser trigger signal, shifting the narrow laser pulse within the sensor modulation waveform as indicated by the dashed arrow on the right hand side of Fig. 3, and sensor integration and readout is repeated. From a large number of phase shifted captures a profile of the complete modulation waveform is built up for each pixel within the image sensor.
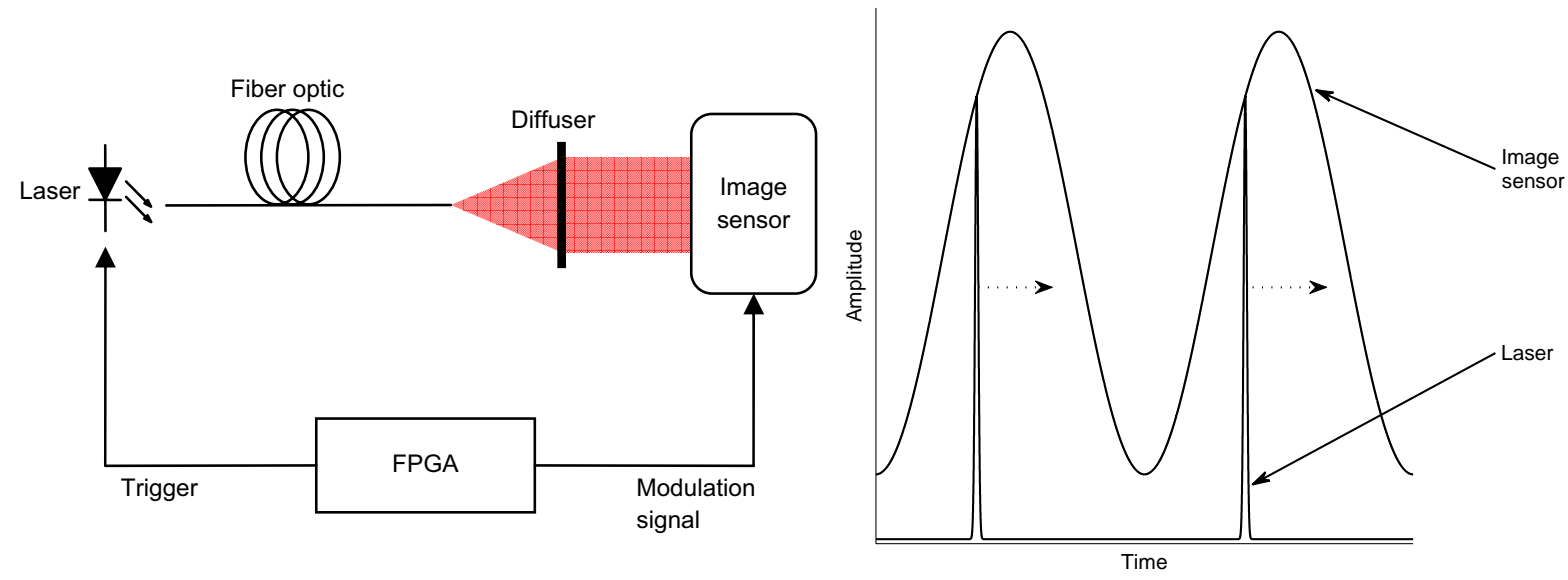

Fig. 3. Left: The configuration used to measure the image sensor modulation response. Right: Diagram showing how a narrow laser pulse is used to optically probe the image sensor modulation waveform. The dashed arrow indicates the incremental phase shift between the laser pulse and the image sensor between captures.

\section{EXPERIMENTAL RESULTS}

A PMD 3K modulated image sensor ${ }^{8}$ was setup as illustrated in the left hand side of Fig. 3 . The sensor uses a custom built PCB which provides the high frequency modulation, control and readout circuitry. A Stratix II FPGA was configured to generate the laser trigger and sensor modulation signals using an internal phase locked loop (PLL), 
producing an output ranging from $5 \mathrm{MHz}$ to $100 \mathrm{MHz}$ with $5 \mathrm{MHz}$ increments. This particular FPGA was selected as the PLL output phase can be varied dynamically, allowing the laser trigger signal phase to be stepped in small increments between captures. A phase step resolution of $4.5^{\circ}$ was chosen, resulting in 80 samples over the sensor modulation period. The laser pulser circuit uses a $658 \mathrm{~nm}$ gain switched laser diode to generate output pulses of less than 300 picosecond duration ${ }^{7}$, illuminating the sensor as described in section 3, while the average optical power was adjusted to be constant for different repetition rates.

The measured voltages from a region of $8 \times 8$ pixels in the center of the image sensor were averaged together to reduce noise, and are shown in Fig. 4 for operating frequencies between 10 and $80 \mathrm{MHz}$. Each measurement was repeated five times at each frequency and the results overlaid on each graph (hence small variations between captures can be seen in Fig. 4 as dark lines). At low frequencies (below $20 \mathrm{MHz}$ ) the modulation waveform is relatively square as the sensor correctly tracks the digital switching modulation input. As the modulation frequency is increased, bandwidth limitations reduce the modulation waveform to a triangular shape (approximately $40-60 \mathrm{MHz}$ ), and then significantly reduce the peak-to-peak amplitude of the waveform (above $70 \mathrm{MHz}$ ).
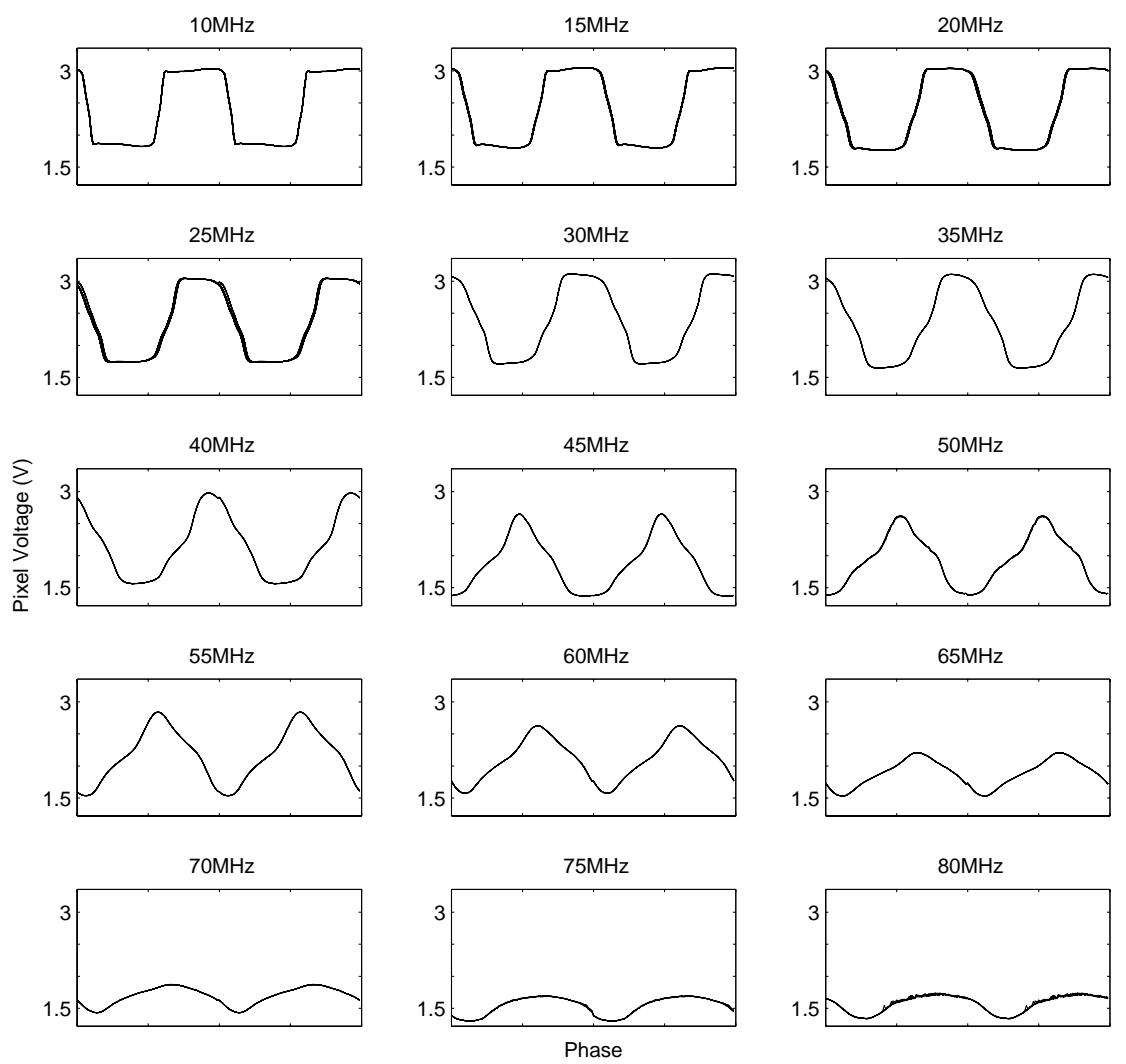

Fig. 4. Measured PMD 3k image sensor modulation waveform at a variety of frequencies.

\subsection{Selecting the optimal modulation frequency}

Range measurement precision is dependent on the sensor modulation frequency $f_{\text {mod }}$ and demodulation contrast $c_{\text {demod }}$ as discussed in section 2.2, where $c_{\text {demod }}$ is proportional to the image sensor modulation depth $m_{\text {Sensor }}$ as given by (7). Therefore, the optimal image sensor operating frequency will maximize both $f_{\bmod }$ and $m_{\text {Sensor }}$ to achieve high range measurement precision. During normal operation the sensor performs a subtraction of the two modulation channels 1 and 2 as illustrated in Fig. 2, removing the offset voltage $A_{\text {sig }}$ (and also ambient illumination $A_{\text {amb }}$ ) from the output. It is therefore not possible to directly calculate the modulation depth $m_{\text {sensor }}$ from the captured data. However, the modulation waveform amplitude $A$ can be measured as an indicator of the modulation depth at each operating frequency to determine the image sensor performance. 
For sinusoidal modulation, the amplitude $A$ is simply calculated as half of the peak-to-peak amplitude of the waveform. From Fig. 4 it is clear that the sensor modulation is not sinusoidal, and simply halving the peak-to-peak amplitude is not sufficient to describe the performance of the sensor. Instead a fast Fourier transform (FFT) is performed on the captured sensor waveform, and the magnitude of the fundamental frequency bin is taken to determine the waveform amplitude $A$. This approach is required as the sensor output is effectively a correlation between the illumination and sensor modulation waveforms, where, for example, square wave modulation produces a larger output than a triangular wave with the same peak-to-peak amplitude. The use of the FFT therefore allows a fair comparison to be made between waveforms of different shapes.

The sensor modulation amplitude $A$ is illustrated in the left hand side of Fig. 5, indicated by a $\times$ marker for each frequency measured. As expected, the amplitude decreases at higher frequencies due to limited sensor (and electronic driver) bandwidth. To find the optimal operating frequency, the amplitude $A$ is multiplied by the modulation frequency $f_{\text {mod }}$ to give a relative range measurement precision value as plotted in the right hand side of Fig. 5, again using $\times$ markers. The precision first rapidly rises with operating frequency as the amplitude $A$ is relatively constant between 10 and $35 \mathrm{MHz}$, and rapidly falls above $55 \mathrm{MHz}$ as the amplitude $A$ decreases. The precision graph peaks at $55 \mathrm{MHz}$, implying that this is the optimal operating frequency of this image sensor and corresponding driver PCB.
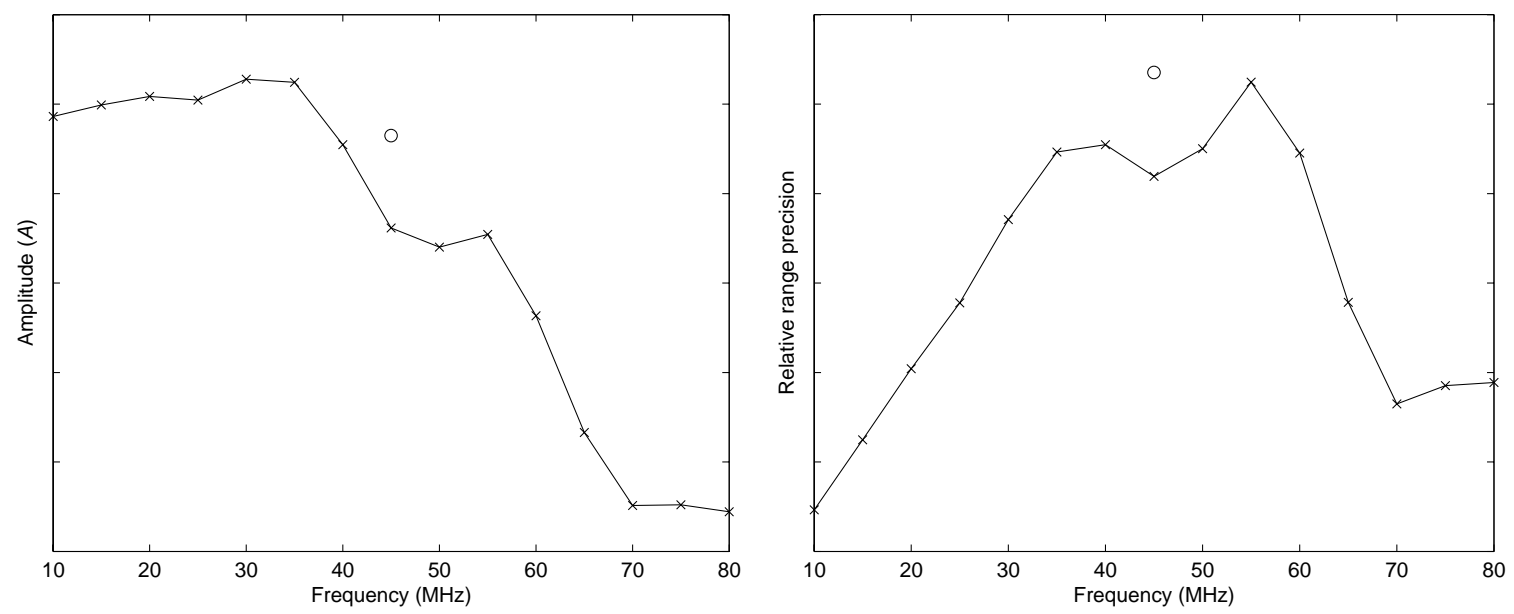

Fig. 5. Left: The measured PMD $3 \mathrm{~K}$ modulation amplitude $A$ taken from data in Fig. 4. Right: The relative range measurement precision calculated by multiplying the measured amplitude $A$ by the modulation frequency $f_{\text {mod. }}$. The circle marker at $45 \mathrm{MHz}$ in each plot is the result of modifying the electronic duty cycle as discussed in section 4.2.

\subsection{Modifying the electronic sensor input}

The relative range precision graph on the right hand side of Fig. 5 has an unexpected deviation around the 40-50 MHz region, where the precision value dips before increasing again at $55 \mathrm{MHz}$. Investigation of the waveforms in Fig. 4 at these frequencies reveal that the modulation is not symmetrical, exhibiting a long flat period where the output is low, and only a brief triangular peak where the output is high. The cause of this asymmetry was established as being due to an imbalance of the electronic path lengths (and associated parasitic inductance) on the PCB controlling the image sensor, resulting in an uneven electronic duty cycle at the sensor. Rather than redesigning the PCB to address this problem, compensation was applied by altering the duty cycle of the high frequency input signal.

The result of this compensation is shown in Fig. 6 for a modulation frequency of $45 \mathrm{MHz}$. The plot on the left hand side is reproduced from Fig. 4 with a standard 50\% electronic input duty cycle, while the plot on the high hand side has the electronic duty cycle reduced to $47.5 \%$. This seemingly small change has a significant impact on the operation of the image sensor which now produces a much more symmetrical waveform with larger amplitude. 

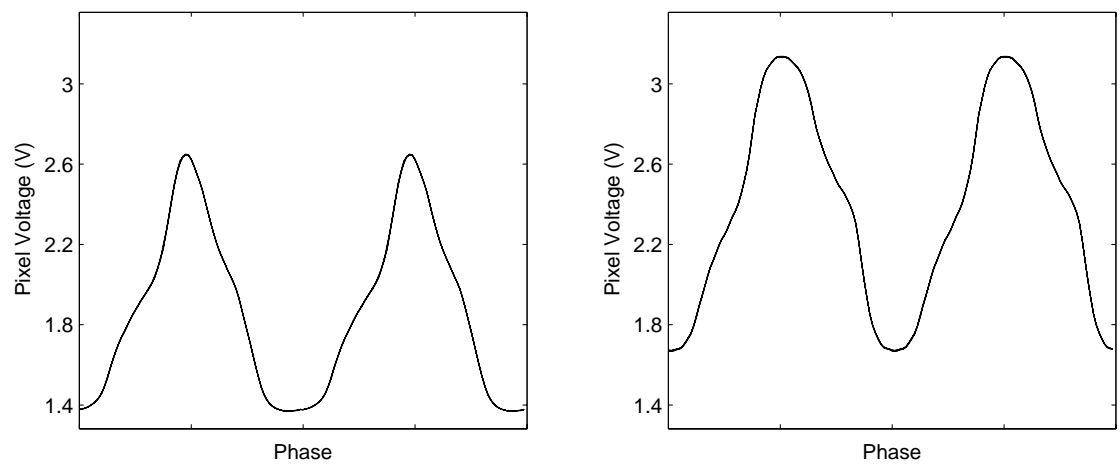

Fig. 6. Left: Measured image sensor modulation waveform at $45 \mathrm{MHz}$ generated using a standard 50\% electrical duty cycle input, Right: The sensor modulation waveform at $45 \mathrm{MHz}$ with the electronic duty cycle input reduced to $47.5 \%$.

The amplitude $A$ of the new $45 \mathrm{MHz}$ waveform was measured using a FFT as described in section 4.1 , resulting in a $22 \%$ improvement over the original response at that frequency. This improvement is illustrated in Fig. 5 by the addition of a circle marker in the amplitude and relative precision plots. Of particular interest, the new $45 \mathrm{MHz}$ waveform now exceeds the maximum peak value at $55 \mathrm{MHz}$ in the right hand plot, providing an increase in precision at a lower modulation frequency.

Use of a lower modulation frequency is significant as it extends the unambiguous distance the system can measure. Object distance is determined by measuring the phase of the received waveform as given by (4), constraining the phase value to fall between 0 and $2 \pi$. If the object distance exceeds $c /\left(2 f_{\text {mod }}\right)$ then the resulting phase shift exceeds $2 \pi$, and the measured phase value wraps back around to fall within the 0 to $2 \pi$ range due to phase being cyclic. This effect creates ambiguity in the range measurement as, for example, objects at $1 \mathrm{~m}$ and $4 \mathrm{~m}$ have the same phase value for a modulation frequency of $50 \mathrm{MHz}$. This ambiguity distance is inversely proportional to the modulation frequency $f_{\text {mod, }}$ so it is desirable to minimize $f_{\text {mod }}$ where possible to allow measurements to be made over larger distances. Alternatively, the ambiguity distance can be extended by using multiple modulation frequencies to measure the scene ${ }^{9}$, allowing high precision measurements to be performed over significantly longer distances.

\subsection{Measuring harmonic content}

Range measurement accuracy is compromised when the illumination and image sensor modulation waveforms are not sinusoidal due to higher frequency harmonics being aliased during sampling as discussed in section 2.3. As the shape of the image sensor modulation waveform has been measured, a fast Fourier transform (FFT) can be performed on the data to determine the harmonic content of the waveform. As an example, the normalized magnitude from the FFT is shown in Fig. 6 for the $45 \mathrm{MHz}, 47.5 \%$ duty cycle waveform illustrated in the right hand side of Fig. 5

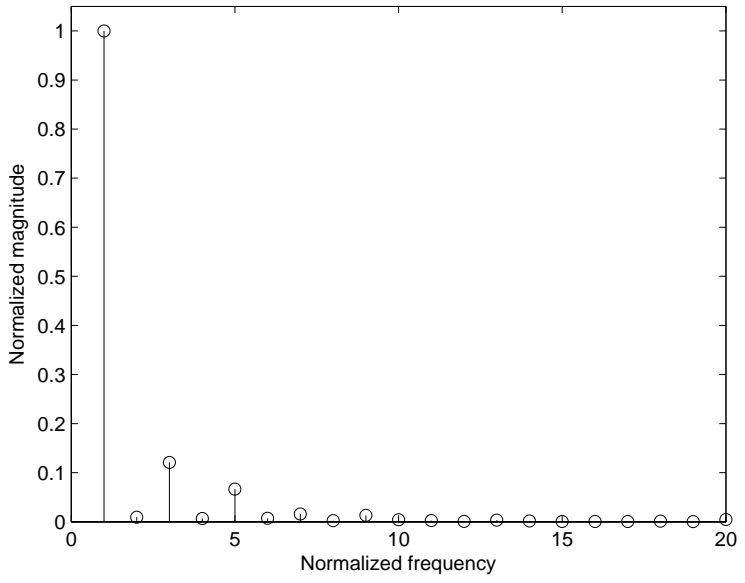

Fig. 7. Harmonic content of the improved image sensor modulation waveform at $45 \mathrm{MHz}$ (using $47.5 \%$ electrical duty cycle). 
The plot in Fig. 6 illustrates a relatively strong presence of third and fifth order harmonics in the image sensor modulation waveform, while harmonics above the tenth order are negligible. Knowledge of this spectrum can be used to create an appropriate control scheme to cancel the effect of these harmonics ${ }^{6}$, improving the measurement linearity, hence accuracy.

\subsection{Spatial offsets}

While the analysis has so far concentrated on the amplitude and temporal shape of the image sensor modulation waveform, spatial variations across the sensor can also be measured from the captured data. Fig. 8 shows an example of these spatial variations, where the phase of the high frequency modulation waveform has independently been calculated for each pixel and converted to an offset distance using (4). In this instance, the modulation frequency used was $40 \mathrm{MHz}$. Two significant effects are visible within the figure: (i) the image sensor is divided into four columns, and (ii) a slope exists within each column. The PMD 3K image sensor is driven by an external source which provides the high frequency modulation voltages to the sensor. To reduce capacitive loading, the image sensor is divided into four columns, each providing one quarter of the total capacitive load. The PCB used to test the sensor during this study only uses a single clock buffer IC which is connected to all four columns; hence the modulation should appear identical across the sensor. However, small variations in the PCB track routing (and corresponding parasitic inductance) add a fixed propagation delay to each column, resulting in the stepped structure shown in Fig. 8.

The slope present within each column is again due to a fixed propagation delay of the modulation voltage reaching each pixel; however in this instance the delay is internal within the sensor rather than external through the PCB routing. The delay is continuous from pixel to pixel as the modulation voltage propagates through the image sensor, resulting in the continuous slope visible in Fig. 8. The combined external and internal propagation delays result in a pixel location dependent offset of up to $3 \mathrm{~cm}$ in this instance, significantly compromising the range system accuracy. The measured pixel offsets are constant for a particular modulation frequency, therefore the resultant range error can be calibrated from these data.

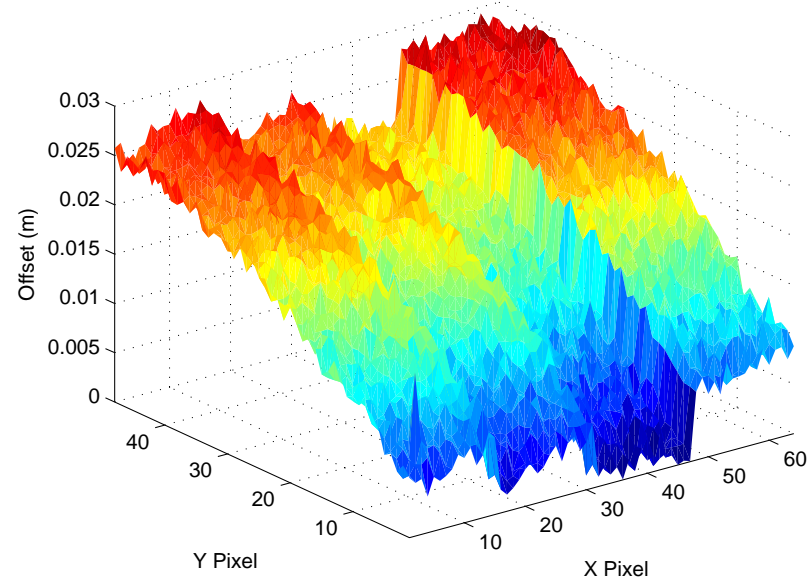

Fig. 8. Measured distance offset for each pixel in the image sensor at a modulation frequency of $40 \mathrm{MHz}$.

\section{EVALUATION AND CONCLUSION}

The ability to measure the high frequency modulation response of time-of-flight image sensors is a powerful tool that allows the image sensor operation to be characterized and optimized independent of other components within a range imaging system. This approach, rather than characterizing the complete system, allows parameters affecting range measurement precision and accuracy to be identified for the sensor itself, and deficiencies to be identified and corrected or calibrated to minimize resulting range measurement errors.

A PMD 3K sensor and custom built driver/control PCB was used during this study to demonstrate the effectiveness of this approach. The sensor modulation waveform was measured over a wide range of operating frequencies, allowing the corresponding amplitude at each frequency to be determined. The results showed that the sensor and driver circuit has a bandwidth exceeding $60 \mathrm{MHz}$, yet typically these sensors are operated at a modulation frequency of $20 \mathrm{MHz}$. The 
optimal operating frequency, determined by multiplying the modulation frequency $f_{\text {mod }}$ by the sensor amplitude $A$, illustrated that the measurement precision could be potentially be doubled, i.e. the standard deviation halved, by operating the sensor at frequencies above $20 \mathrm{MHz}$. In practice, however, the illumination source also has a corresponding limited bandwidth which must be taken into account when selecting the modulation frequency for the system. By independently measuring the temporal response of the image sensor and illumination source, limiting components within the system can readily be identified and addressed. For example, if the modulation frequency for a given system is constrained by the bandwidth of LEDs used as the illumination source, these could potentially be replaced with laser diodes to provide a significant increase in modulation speed, providing a corresponding increase in measurement precision.

The shape of the image sensor modulation waveform is also of interest, in this instance allowing an issue related to the sensor modulation duty cycle to be identified. Compensation was applied to the hardware by adjusting the duty cycle of the input signal slightly, resulting in a $22 \%$ increase in the resulting modulation amplitude at the $45 \mathrm{MHz}$ operating frequency. Similar compensation could be applied at other operating frequencies, for example at $40 \mathrm{and} 50 \mathrm{MHz}$, where the duty cycle shows a similar, although less pronounced, offset. The sensor modulation waveform shape also contributes towards range measurement linearity errors due to harmonics being aliased during sampling, reducing range measurement accuracy. As the image sensor modulation waveform shape is now known, the harmonics can be identified by performing a FFT on the captured data and cancelled from the waveform using a modified modulation technique ${ }^{6}$.

The data also allow pixel location dependent delays to be measured to determine the corresponding resultant offset in the range measurement. In this instance the propagation delays were both internal and external from the sensor. It is unlikely that these delays can be removed completely from the hardware as they are due to the propagation delay of the high frequency signals, however by measuring the resulting range offset, compensation can be applied through post processing of the range data.

The example characterization of the PMD 3K image sensor demonstrates the effectiveness of examining the sensor independently from the range imaging system, allowing the optimal operating conditions such as the modulation frequency to be determined. Underlying parameters affecting range measurement performance can then be investigated from the captured data, and corrections or compensation applied to mitigate resulting errors, allowing the range imaging sensor to measure distances with improved precision and accuracy.

The sensor characterization method can also be used to evaluate and compare the performance of different modulated image sensors. A number of range imaging systems are available from various manufacturers including PMD Technologies $\mathrm{GmbH}^{8}$, Canesta Inc. ${ }^{10}$, and MESA Imaging ${ }^{11}$, which measure distance in the same manner as the PMD 3K image sensor used during this study, i.e. by modulating each pixel within the image sensor at high frequency. The internal pixel design varies significantly between different manufacturers; therefore the characterization technique demonstrated could be used as a tool to evaluate the performance of each of the different sensors independent of the illumination source used within each range imaging system.

\section{ACKNOWLEGEMENTS}

The authors would like to thank Richard Conroy and Adrian Jongenelen for their contribution towards the setup of the PMD sensor and the FPGA code used to control the sensor and laser. Funding was provided for this work through the University of Waikato strategic investment fund.

\section{REFERENCES}

[1] Kahlmann, T. and Ingensand, H., "Calibration of the fast range imaging camera SwissRanger for use in the surveillance of the environment," Proc. SPIE 6396, 639605 (2006).

[2] Büttgen, B., Oggier, T., Lehmann, M., Kaufmann, R. and Lustenberger, F., "CCD/CMOS Lock-In Pixel for Range Imaging: Challenges, Limitations and State-of-the-Art," 1st Range Imaging Research Day, 21-32 (2005).

[3] Dorrington, A. A., Cree, M. J., Carnegie, D. A., Payne, A. D. and Conroy, R. M., "Heterodyne range imaging as an alternative to photogrammetry," Proc. SPIE 6491, 64910D (2007). 
Mure-Dubois J. and Hugli, H., "Optimized scattering compensation for time-of-flight camera," Proc. SPIE 6762, $67620 \mathrm{H} \mathrm{(2007).}$

[5] Lange, R., "3D Time-of-flight distance measurement with custom solid-state image sensors in CMOS/CCDtechnology," Ph.D. dissertation, University of Siegen (2000).

[6] Payne, A. D., Dorrington, A. A., Cree, M. J. and Carnegie, D. A. "Improved Linearity Using Harmonic Error Rejection in a Full-Field Range Imaging System," Proc. SPIE 6805, 68050D (2008).

[7] Payne, A. D., Dorrington, A. A., Cree, M. J. and Carnegie, D. A. "Characterizing an Image Intensifier in a Full-Field Range Imaging System," IEEE Sensors Journal, 8(11), 1763-1770, (2008).

[8] PMD Technologies GmbH, http://www.pmdtec.com/e_index.htm

[9] Dorrington, A. A., Cree, M. J., Payne, A. D. and Conroy, R. M., Carnegie, D. A., "Achieving sub-millimetre precision with a solid-state full-field heterodyning range imaging camera," Meas. Sci. Technol., 18, 2809-2816 (2007).

[10] Canesta Inc., http://canesta.com/

[11] MESA Imaging, http://www.mesa-imaging.ch/ 